Journal of Animal and Veterinary Advances 11 (15): 2623-2629, 2012

ISSN: $1680-5593$

(C) Medwell Journals, 2012

\title{
Interaction Between VirB5 of Brucella Type IV Secretion System (TFSS) and Ferritin Heavy Polypeptide 1 (FTH1) in Murine Macrophage
}

\author{
${ }^{1}$ Fei Guo, ${ }^{3}$ Yuanzhi Wang, ${ }^{1}$ Chuangfu Chen, ${ }^{2}$ Hui Zhang, ${ }^{1}$ Jun Qiao, \\ ${ }^{3}$ Yan Ren, ${ }^{1}$ Junbo Zhang and ${ }^{1}$ Zhiqiang Li \\ ${ }^{1}$ College of Animal Science and Technology, \\ ${ }^{2}$ Key Laboratory of Xijiang Endemic and Ethnic Disease, \\ ${ }^{3}$ College of Medicine, Shihezi University, 832003 Shihezi, China
}

\begin{abstract}
TFSS is an important virulence factor of Brucella, organized as one operon containing 12 different across the cell wall bacterial proteins among which VirB5 regulates the host phagocytosis of Brucella and the transportation of Brucella in the host cells. This study has constructed cDNA library from Brucella melitensis 16M-infected murine macrophage Raw264.7, identified and confirmed the interaction between Brucella VirB5 and FTH1 of RAW264.7 using yeast two-hybrid and Co-Immunoprecipitation (Co-IP) technologies. Subsequently, the morphological changes and the expression of apoptosis-related genes in Brucella-infected RAW264.7 cells have been investigated with Electron Microscope (EM) and real-time quantitative RT-PCR, respectively. The present study has demonstrated that VirB5 and FTH1 play important roles in intracellular parasitism of Brucella and inhibition of FHT1 expression accelerates the apoptosis of macrophage.
\end{abstract}

Key words: Yeast two-hybrid, cDNA library, FTH1, Brucella, co-immunoprecipitation

\section{INTRODUCTION}

Brucellosis is a zoonotic disease (Pappas et al., 2006; Delrue et al., 2001) caused by facultative intracellular parasitic bacteria Brucella. Brucella infection in human induces bone and joint damages as well as damages in nervous, reproductive, circulatory and immune systems and is often accompanied by bacteremia. Brucella infections in animal often cause abortion, premature birth, stillbirth, orchitis and epididymitis among other diseases and bring serious harm to human health and the development of animal husbandry (Rajashekara et al., 2006; Guerra, 2007). In addition, Brucella is also an important pathogen of bioterrorism (Robinson-Dunn, 2002). Therefore to understand the pathogenesis of Brucella infecting host cells becomes a hot topic in the field in recent years.

TFSS proteins directly relate to the intracellular survival and reproduction of Brucella and are well recognized as its virulent factors (Boschiroli et al., 2002; O'Callaghan et al., 1999). TFSS is a complex of multiprotein family (Boschiroli et al., 2002; O'Callaghan et al., 1999) comprised of 12 different across-the-cell-wall proteins which are organized in one operon and indispensible for the intracellular replication and persistent infection of bacteria in the host. VirB12 as an antigenic marker for serological detection of Brucella can be used in the study of its infection process in laboratory animals and natural host (Rolan et al., 2008); VirB1 is a non-essential component of TFSS; VirB3, 6, 7, 8, 9 and 10 are distributed in the membrane pores to transduce the signals; VirB4 and 11 are two ATPase molecules and the former has a complete ATP binding domain and VirB5 is a bacterial surface protein with pilus-like structure and can regulate the host phagocytosis and intracellular transportation of Brucella (Rouot et al., 2003).

The molecular mechanism of the intracellular parasitism of Brucella is closely related to the binding between its virulent factors and the targeting proteins in macrophage. However, few reports about macrophage proteins interacting with TFSS of Brucella are currently available. This study has demonstrated the interaction between VirB5 of Brucella TFSS and FTH1 in macrophage and confirmed that FTH1 plays an important role in the intracellular parasitism of Brucella and inhibition of FTH1 expression in macrophage accelerates the cell apoptosis.

\section{MATERIALS AND METHODS}

Bacterial strains: Saccharomyces cerevisiae strains AH109 (MATa) and Y187 were purchased from Clontech (USA). Brucella melitensis $16 \mathrm{M}$ and E. coli Top1 0 were passaged in the laboratory.

Corresponding Author: Chuangfu Chen, College of Animal Science and Technology, Shihezi University, 832003 Shihezi, China 
Major reagents: The TRizol reagent for RNA extraction was purchased from Invitrogen (USA); Matchmaker ${ }^{\mathrm{TM}}$ Library Construction and Screening Kit, Matchmaker ${ }^{\mathrm{TM}}$ Co-IP Kit, Advantage 2 PCR Kit, YPD medium and SD/Leu selective medium were all purchased from Clontech (USA). Other immunoprecipitation reagents, TNT T7 Coupled Reticulocyte Lysate System, RNasin Ribonuclease Inhibitor and Transcend ${ }^{\mathrm{TM}}$ Non-Radioactive Translation Detection Systems were provided by Promega (USA).

cDNA library construction from Brucella melitensis 16M-infected RAW264.7 cells: The cDNA library was constructed as described before (Robinson-Dunn, 2002).

Construction of the pGBKT7-virB5 bait system: PCR primers for cloning the virB5 of Brucella melitensis $16 \mathrm{M}$ were designed as the following: P1:5'-GAATTCATGA AGAAGATAATTCTCAGC-3'; P2:5'-CTGCAGTTAAT AGGCGGCTTCCA-3'. The PCR product was digested and cloned into pGBKT7 at EcoR I/Pst I site. The recombinant plasmid was transformed into yeast strain Y187 and verified by self-activation.

The negative and positive controls of yeast two-hybrid system: While the competent $\mathrm{AH} 109$ cells transformed with pGADT7-T from the Matchmaker ${ }^{\mathrm{TM}}$ Library Construction and Screening Kit were used as control for the prey system, the pGBKT7-53 and pGBKT7-Lam transformed yeast Y187 cells were used as positive and negative controls, respectively for the bait system. The pre-two-hybrid experiments were then performed to test the positive and negative control systems.

Interactive screening of yeast strains Y187 (pGBKT7virB5) and AH109 (pGADT7-cDNA liberary): pGBKT7virB5-containing yeast $\mathrm{Y} 187$ was inoculated into $50 \mathrm{~mL}$ $\mathrm{SD} /-\operatorname{Trp} / \mathrm{Kan}\left(50 \mu \mathrm{g} \mathrm{mL} \mathrm{m}^{-1}\right)$ liquid medium and cultivated for $20 \mathrm{~h}$ at $30^{\circ} \mathrm{C}$ with $40 \mathrm{r} \mathrm{min}^{-1}$ shaking. The bacterial density was then concentrated to $>1 \times 10^{9}$ cells $\mathrm{mL}^{-1}$ by centrifugation. Yeast AH109 (pGADT7-cDNA library) culture with a density $>2 \times 10^{7}$ cells $\mathrm{mL}^{-1}$ was removed from $-80^{\circ} \mathrm{C}$ refrigerator and melted. About $45 \mathrm{~mL}$ of $2 \times \mathrm{YPD} / \mathrm{Kan}\left(50 \mu \mathrm{g} \mathrm{mL}^{-1}\right)$ medium, $1 \mathrm{~mL}$ of yeast AH109 (pGADT7-cDNA liberary) and $5 \mathrm{~mL}$ of Y187 (pGBKT7virB5) were successively added into sterilized $2 \mathrm{~L}$ culture flask and cultivated for $20 \mathrm{~h}$ at $30^{\circ} \mathrm{C}$ with $40 \mathrm{r} \mathrm{min}^{-1}$ shaking. The culture was harvested by centrifugation, washed twice with $50 \mathrm{~mL} 0.5 \times \mathrm{YPD} / \mathrm{Kan}\left(50 \mu \mathrm{g} \mathrm{mL}^{-1}\right)$ and re-suspended in $1 \mathrm{~mL} 0.5 \times \mathrm{YPD} / \operatorname{Kan}\left(50 \mu \mathrm{g} \mathrm{mL}{ }^{-1}\right)$. The re-suspended yeast culture was spread onto 40 of $150 \mathrm{~mm}$ $\mathrm{SD} /$-Ade/-His/-Leu/-Trp plates which were then inverted and cultivated at $30^{\circ} \mathrm{C}$ for 4 days. The grown colonies were screened with $\mathrm{X}-\alpha-\mathrm{Gal}$.

Extraction and sequencing of the captured plasmids: Plasmids were extracted by kit from the positive colonies which were identified from the SD/-Ade/-His/-Leu/-Trp/X$\alpha$-Gal screening, PCR amplified and sequenced.

Validation of the yeast two-hybrid results by co-IP: pGBKT7-virB5 and the positive plasmids from pGADT7cDNA library screening were in vitro transcribed and translated with TNT T7 Coupled Reticulocyte Lysate System Kit. About $4 \mu \mathrm{L}$ of respective in vitro transcribed and translated bait and prey proteins were added into eppendorff tube incubated at room temperature for $1 \mathrm{~h}$, separated by SDS-PAGE, blotted onto nitrocellulose membrane and stained with Western Blue Stabilized Substrate.

Construction of shRNA-expressing vector targeting the FTH1: Three pairs of shRNAs (targeting the nucleotides $531-552,638-659$ and 705-726, respectively) and one negative control shRNA were designed according to murine FTH1 gene sequence (GenBank accession: NM_010239; Table 1), cloned into RNAi-Ready pSIRENRetroQ ZsGreen Vector and named as pSI-A, pSI-B, pSI-C and pSI-D (negative control), respectively.

Detection of the knock-down effect of FTH1-targeting shRNAs: pSI-A, pSI-B, pSI-C and pSI-D were transfected into macrophages using TurboFect ${ }^{\mathrm{TM}}$ in vitro transfection reagent. The transfection efficiency was confirmed with a confocal microscope $48 \mathrm{~h}$

Table 1: Sequences of the synthetic shRNAs

\begin{tabular}{|c|c|}
\hline shRNA & Sequences \\
\hline FTH1-A1 & 5'-GATCCAAACTGATGAAGCTGCAGAACTTCAAGAGAGTTCTGCAGCTTCATCAGTTTTTTTTTACGCGTG-3' \\
\hline FTH1-A2 & 5'-AATTCACGCGTAAAAAAAAACTGATGAAGCTGCAGAACTCTCTTGAAGTTCTGCAGCTTCATCAGTTTG-3 \\
\hline FTH1-B1 & 5'-GATCCAAA GAAACCAGACCGTGATGATTCAAGAGATCATCACGGTCTGGTTTCTTTTTTTTTACGCGTG-3' \\
\hline FTH1-B2 & 5'-AATTCACGCGTAAAAAAAAAGAAACCAGACCGTGATGATCTCTTGAATCATCACGGTCTGGTTTCTTTG-3 \\
\hline FTH1-C1 & 5'-GATCCAATCAGTCACTACTGGAACTGTTCAAGAGACA GTTCCAGTAGTGACTGATTTTTTTTACGCGTG-3' \\
\hline FTH1-C2 & 5'-AATTCACGCGTAAAAAAAATCAGTCACTACTGGAACTCTCTCTTGAACAGTTCCAGTAGTGACTGATTG-3 \\
\hline FTH1-D1 & 5'-GATCCAATTCGAATCTCGCTGACCAGTTCAAGAGACTGGTCAGCGAGATTCGAATTTTTTTTACGCGTG-3' \\
\hline FTH1-D2 & 5'-AATTCACGCGTAAAAAAAATTCGAATCTCGCTGACCAGTCTCTTGAACTGGTCAGCGAGATTCGAATTG-’’ \\
\hline
\end{tabular}


Table 2: Primer sequences for FTH1, GAPDH and apoptosis related genes

\begin{tabular}{ll}
\hline Primer names & Sequences \\
\hline FTH1-s & GCAGGATATAAAGAAACCAGA \\
FTH1-A & TCTCAATGAAGTCACATAAGT \\
GAPDH-S & GTCGTGGAGTCTACTGGTGT \\
GAPDH-A & TGCTGACAATCTTGAGTGA \\
16 r rRNA-s & CACCCCGACGGCTAACATTCA \\
16 rRNA-A & AGTGTAGAGGTGAAATCGTA \\
Mkl1-s & TGGAGCTGGTGGAGAAGAATA \\
Mkl1-A & CCCTGGGACTCATGGCTGGCA \\
Birclb-s & GACAGTTTCCAGTAGCCAGTA \\
Birc1b-A & CCACCAACTATAGATCAGGAC \\
Hip1-S & CCTCCCAGCAGACTTTTTT \\
Hip1-A & CATGTTGTCCAGCTGCCCTGT \\
Taf10-S & GGAGAAGTGAAGCCCGTAGTG \\
Taf10-A & AGATGAGCCGATTATGCGTG \\
Bcl21-S & CGTATCAGAGCTTTGAGCAGG \\
Bcl21-A & AGATAGGTGGCCATCCAACTT \\
Myc-S & GGGCTCTGCTCTCCATCCTAT \\
Myc-A & GATCGCAGATGAAGCTCTGGT \\
AW2600635-S & AAACTGGGGATGAAGGAAATG \\
AW2600635-A & CTCTTCCATATATGCCAGCTT \\
Tnfrsf5-S & CTGGCTTTGGAGTTATGGAGA \\
Tnfrs5-A & TTTCTGTAGGACCTCCAAGT \\
\hline
\end{tabular}

after the transfection. Total RNAs were extracted from transfected cells and the knock-down effects of the shRNAs were evaluated by real-time quantitative RT-PCR.

The expressions of 16S rRNA and apoptosis-related genes in Brucella-infected pSIREN-siRNA-transfected RAW264.7 cells: RAW264.7 cells were transfected with pSI-C and pSI-D (negative control) plasmids. About $48 \mathrm{~h}$ later, the transfected cells were infected with Brucella melitensis $16 \mathrm{M}$. The expressions of $16 \mathrm{~S}$ rRNA, GAPDH and apoptosis-related genes were then detected by real-time quantitative RT-PCR (Table 2). Normalization was done with GAPDH as the internal control. The gene inhibitory effects were calculated using the following equation:

$$
\begin{aligned}
& \text { Interfere efficiency }(\%)= \\
& \frac{\text { Gene expressionin RNAi knock }- \text { Down group }}{\text { Gene expressioninRNAinegativecontrolgroup }} \times 100
\end{aligned}
$$

Real-time quantitative RT-PCR detection was done in triplicates and the data were then statistically analyzed.

EM observation of Brucella melitensis 16M-infected macrophage cells: Brucella melitensis $16 \mathrm{M}$ infecting shRNA vector-transfected RAW264.7 cells was carried out as described above. About $4 \mathrm{~h}$ after the infection, cells were fixed with $2.5 \%$ glutaraldehyde, processed according to the conventional procedures and observed under EM.

\section{RESULTS AND DISCUSSION}

The quality of cDNA library from Brucella melitensis 16M-infected mouse macrophage RAW264.7 cells: The

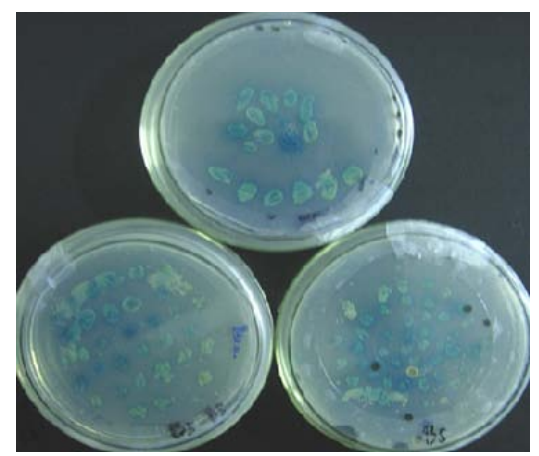

Fig. 1: Positive colonies captured by bait plasmid pGBKT7-virB5 in yeast Y187

complexity of cDNA library is $1.364 \times 10^{6}$ with a recombination rate of $95.65 \%$. The insert size distribution is as the following: $8.5 \%$ inserts are $<500 \mathrm{bp} ; 70 \%$ between $500-1000$ bp; $13 \%$ between $1000-1500$ bp and $8.5 \%$ $>1500$ bp.

Evaluation of the pGBKT7-virB5 bait system: Yeast Y187 (pGBKT7-virB5) is spread onto SD/-Trp and SD/-Trp-His plates, respectively. After culture at $30^{\circ} \mathrm{C}$ for $4-5$ days, 40-100 of 2-3 mm colonies are grown on $\mathrm{SD} /$-Trp plate whereas no colonies grown on SD/-Trp-His plate. When colonies from $\mathrm{SD} /$-Trp plate are transferred onto $\mathrm{SD} /$ $\operatorname{Trp} / \mathrm{X}-\alpha-\mathrm{Gal}$ plate, no self-activations can be observed.

Evaluation of yeast two-hybrid system with positive and negative controls: Yeast strains $\mathrm{AH} 109$ (pGADT7-T) and Y187 (pGBKT7-53) grow into 2-3 mm blue colonies on $\mathrm{SD} / \mathrm{Ade} / \mathrm{-His} / \mathrm{Leu} / \mathrm{Trp} / \mathrm{X}-\alpha$-Gal plate after mixing whereas no colonies are grown on $\mathrm{SD} /-\mathrm{Ade} / \mathrm{-His} / \mathrm{L} \mathrm{Le} /-\mathrm{Trp} / \mathrm{X}-\alpha-$ Gal plate spread with mixed yeast strains of AH109 (pGADT7-T) and Y187 (pGBKT7-1 am).

Interaction between yeast stains Y187 (pGBKT7-virB5) and AH109 (pGADT7-cDNA library): About 2-3 mm colonies are grown on $\mathrm{SD} / \mathrm{Ade} / \mathrm{-His} / \mathrm{Leu} / \mathrm{-Trp}$ plates after spreading of mixed yeast strains of AH109 (pGADT7liberary) and Y187 (pGBKT7-virB5) and some of them grow into blue colonies after transferring onto $\mathrm{SD} / \mathrm{Ade} /-$ His/-Leu/-Trp/X- $\alpha$-Gal plates (Fig. 1). cDNA plasmids from positive blue colonies have been isolated and sequenced. Three genes have been identified and they are purine nucleoside phosphorylase, FTH1 and 26S proteasome non-ATPase regulatory subunit 8 .

Verification of the interactions between VirB5 and the prey proteins by co-IP: The results show that VirB5 protein of Brucella TFSS interacts with macrophage protein FTH1 but does not interact with purine nucleotide phosphodiesterase and $26 \mathrm{~S}$ proteasome non-ATPase regulatory subunit 8 (Fig. 2 ). 
Observation of the transfection efficiency of RAW264.7 cells with confocal microscope: The transfection is done with TurboFect ${ }^{\mathrm{TM}}$ in vitro Transfection Reagent Kit according to manufacturer's instructions. About $48 \mathrm{~h}$ after the transfection, expression of green fluorescent protein is observed under LSM510 laser confocal microscope. based information collected from various fields (Fig. 3).

Fig. 2: Varification of the interaction between VirB5 and FTH1 by co-IP. 1: FTH1; 2: VirB5 and 3: The interaction between VirB5 and FTH1

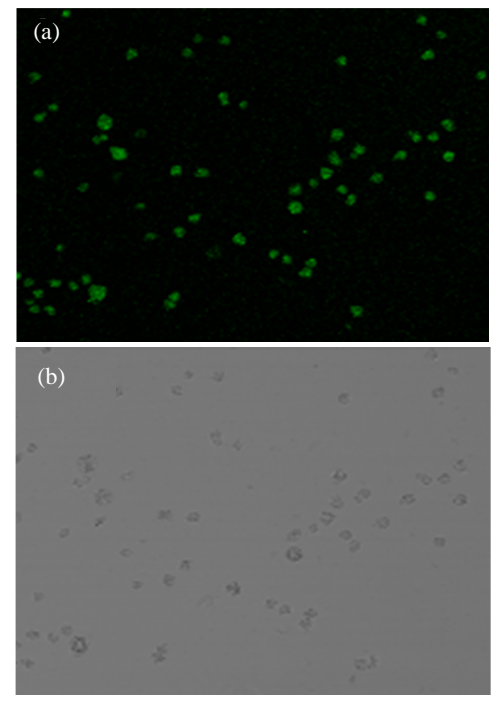

Fig. 3: Observation of RAW264.7 cells under confocal microscope $48 \mathrm{~h}$ after transfection with pSIRENsiRNA plasmids; a) Fluorenscence microscopic photograph and b) light microscopic photograph The calculated transfection efficiency is close to $100 \%$

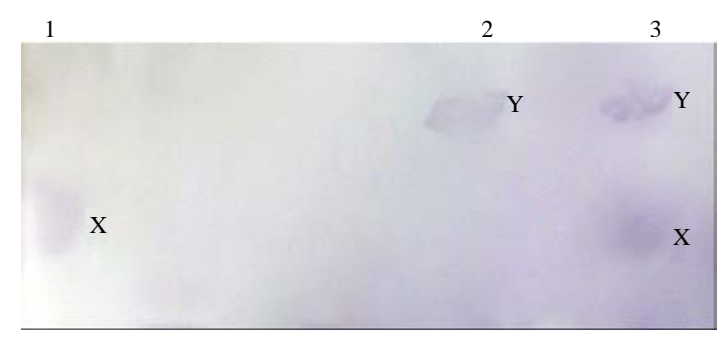

Detection of the knock-down effect of pSIREN-siRNA plasmid: About $48 \mathrm{~h}$ after the transfection of cells with pSI-A, pSI-B, pSI-C and pSI-D plasmids, respectively copy numbers of FTH1 have been detected by real-time quantitative RT-PCR with GAPDH gene as the internal control to calculate the interference efficiency. The results demonstrate that all three experimental RNAi vectors show knock-down effect. pSI-C shows the highest interfere efficiency of $97.60 \%$ (Table 3).

Expression of 16S rRNA and apoptosis-related genes after knock-down of FTH1 in Brucella-infected RAW264.7 cells: Real-time quantitative RT-PCR results show that the expression of $16 \mathrm{~S}$ rRNA is reduced by $84 \%$ in FTH1 knock-down RAW264.7 cells compared to that in cells transfected with control vector while the expressions of Bcl21, Mk11, Tnfrsf5, Myc and Birclb mRNAs are reduced too. However, the expressions of other apoptotic factors show a trend of increase. Among the reduced genes, Mkll and Birclb expressions reduce by $\sim 76.29$ and $\sim 88.85 \%$, respectively (Table 4 ).

EM observation of the morphological changes in Brucella-infected RAW264.7 cells: It can be observed that bacteria are inside the RAW264.7 macrophage cells $4 \mathrm{~h}$ after the infection with Brucella melitensis $16 \mathrm{M}$ at a MOI (Multiplicities of Infection) of 50. EM results indicate that the cells show homogeneous cytoplasm and clear nuclei (Fig. 4b); there are vacuolization of the nuclei and formation of Brandt bodies in the cytoplasm and the heterogeneity of nuclei is intensified (Fig. 4a-c); c and e shows no evident changes in the nucleolus whereas $\mathrm{d}$ shows nucleolar shrinkage and disappearance (Fig. 4a-e). Protein-protein interactions regulate a large number of cellular activities, such as cell proliferation, differentiation

Table 3: Screening of the siRNA with highest interference efficiency

\begin{tabular}{lllll}
\hline Detection & pSIREN-A & pSIREN-B & pSIREN-C & pSIREN-D \\
\hline Average Ct of GAPDH & 22.94 & 26.84 & 25.39 & 30.33 \\
GAPDH copy number & 10969833 & 1521248 & 3171027 & 259657 \\
Average Ct of FTH1 & 24.59 & 30.36 & 31.91 & 29.5 \\
FTH1 copy number & 4188514 & 122247.5 & 47308.59 & 207014.1 \\
Inhibitory rate & 57.60 & 88.50 & 97.60 & - \\
of FTH1 (\%) & & & & \\
\hline
\end{tabular}

Table 4: The expressions of 16S rRNA and apoptosis-related genes

\begin{tabular}{lcccc}
\hline Genes & $\begin{array}{c}\text { Average Ct in } \\
\text { treated group }\end{array}$ & $\begin{array}{c}\text { Average Ct in } \\
\text { control group }\end{array}$ & $\begin{array}{c}\text { Copy number } \\
\text { in treated group }\end{array}$ & $\begin{array}{c}\text { Copy number } \\
\text { in control group }\end{array}$ \\
\hline I6S rRNA & 23.56 & 20.44 & 16684761 & 85838081 \\
Bcl21 & 26.59 & 26.26 & 3250.424 & 3882.934 \\
Mkll & 25.39 & 23.32 & 6125.055 & 20261.89 \\
Tnfrsf5 & 31.90 & 29.19 & 252.9880 & 34.00 \\
AW2600635 & 44.23 & 46.56 & 248.1818 & 7875.038 \\
Birclb & 29.61 & 26.97 & 2148.770 & 84.41896 \\
Hipl & 26.80 & 28.34 & 40738.03 & 15122.72 \\
Taflo & 24.23 & 25.88 & 18158.92 & 23933.16 \\
Myc & 28.93 & 28.69 & 763.6423 & 7962.327 \\
GAPDH & 22.23 & 22.71 & 15718098 & 86.71 \\
\end{tabular}


and death. Protein interactions can be detected with a variety of technologies such as yeast two-hybrid, phage display, co-IP, immunoaffinity chromatography, crosslinking, Fluorescence Resonance Energy Transfer (FRET), external gene suppressor, synthetic lethal screening, etc. Yeast two-hybrid system shows high detection sensitivity and has been widely used in the discovery of unknown proteins that interact with known target proteins. A large number of interactive proteins including many novel ones have been identified using this system. Nonetheless, yeast two-hybrid system has its own drawbacks. For example when specific post-translational modifications of certain protein fail to be done in yeast cells or protein fusion changes the intrinsic activity and folding of a protein, false-negative results are unavoidable. This study has used co-IP to further verify the results from yeast two-hybrid screening. Co-IP can detect protein complex formed in vivo in their naturally modified state and to exclude the false-positive results introduced by overexpression of the target protein. This study has identified and confirmed the interaction between Brucella VirB5 and FTH1.
Ferritin regulates iron storage and maintains iron homeostasis in the cells. Ferritin protein consists of heavy and light chains (Orino and Watanabe, 2008). Tsuji et al. (1999) demonstrate that FTH gene has Antioxidant Response Elements (AREs) upstream its transcription start site, responses to oxidative stress and protects cells from oxidative damages (Tsuji et al., 1999; Tsuji, 2005). Extensive evidences show that the virulence of pathogenic bacteria directly relates to their iron-uptake ability. Effective iron uptake itself is considered as an important virulence factor and pathogenic bacteria must employ a highly specific and effective iron-uptake system to achieve this function (Mikhael et al., 2006). Pham et al. (2004) believe that ferritin plays an important role in inhibition of iron-mediated oxidative damages. Activation of nuclear transcription factors such as NF- $\mathrm{kB}$ and acceleration of the expression of apoptosis-related genes constitute one of the mechanisms by which oxidative damage induces cell apoptosis. This study has demonstrated the interaction between macrophage FTH1 and Brucella VirB5 and the researchers speculate that activated TFSS of Brucella transports chelated iron into

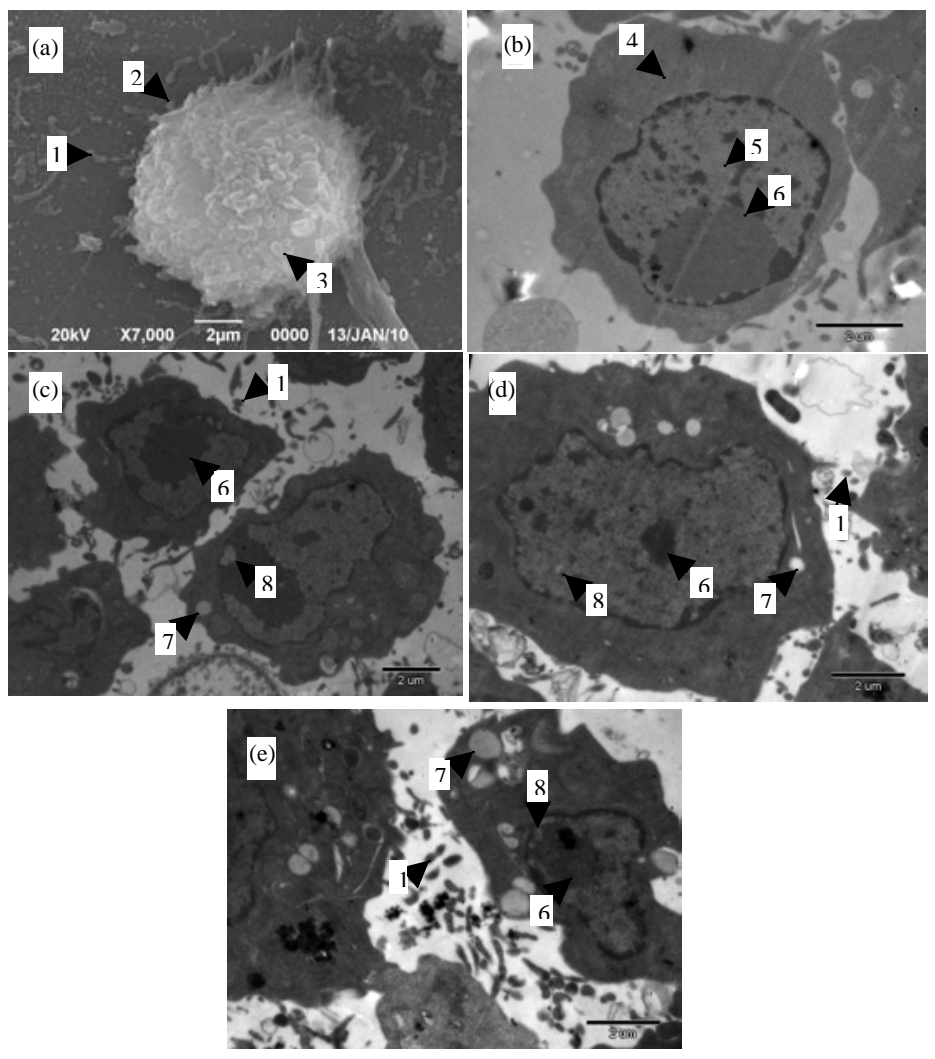

Fig. 4: a-e) Morphology of RAW264.7 cells under EM; 1: Free Brucella bacterium; 2: Tethered Brucella bacterium; 3: Invading Brucella bacterium; 4: Cytoplasm; 5: Nucleus; 6: Nucleolus; 7: Brucellosome and 8: Nuclear vacuolization 
the bacterium body to provide the necessary iron to bacteria, hence maintaining the bacterial virulence. On the other hand, FTH1 chelating cytosolic free iron in infected cells reduces the divalent iron ion-caused cell injuries, plays a role of anti-oxidation, attenuates cell apoptosis and hence promotes the long-term survival of Brucella in macrophages.

The occurrence of cell apoptosis is related to oxidative damage, calcium overload and mitochondria damage, among other factors. At the molecular level, cell apoptosis is regulated by three types of genes: anti-apoptotic genes, apoptotic genes and two-way regulatory genes. Studies from (He et al., 2006) show that 24-48 $\mathrm{h}$ after the infection of murine J774.A1 macrophage cells with Brucella melitensis $16 \mathrm{M}$, the transcription of anti-apoptotic genes Mkll and Birclb increases significantly whereas the expression of mitochondrial type I and III genes decreases, resulting in the Reduction in ROS (reactive oxygen species). This prevents cytochrome $\mathrm{c}$ release and mitochondrial production of ROS and free radicals and inhibits the early apoptotic signal transduction. This study has designed three pairs of siRNAs targeting the FTH1 gene. Among them, the one with best knock-down effect has been selected by real-time quantitative RT-PCR and transferred into RAW264.7 macrophage cells to study the effect of FTH1 interference on the expressions of $16 \mathrm{~S}$ rRNA and apoptosis-related genes.

\section{CONCLUSION}

The results show that expression of $16 \mathrm{~S}$ rRNA in siRNA-transfected cells reduces by $83 \%$ compared to that in control group. The expressions of apoptosis-related genes Bcl21, Mk11, Tnfrsf5, AW2600635 and Birclb decline too; among them, Mkll and Birclb mRNA expressions show the greatest changes and are reduced by $\sim 76.3$ and $\sim 88.8 \%$, respectively. Expressions of rest the apoptosis-related genes show an insignificant trend of increase. Relevant data indicate that both Mkll and Birclb are anti-apoptotic genes (Mikhael et al., 2006). EM results show that compared to the normal control group, shRNAinterfered RAW264.7 macrophages after Brucella infection exhibit apoptotic characteristics such as nucleolar shrinkage and disappearance which is consistent with the results of real-time quantitative detection indicating that FTH1 plays an important role in intracellular parasitism of Brucella.

Inhibition of FTH1 in macrophages accelerates the cell apoptosis and after collapse of the macrophage cells, once again, Brucella bacteria have to face the adverse environment of antibodies, complements and other factors which eventually impede their parasitism and transmission.

\section{REFERENCES}

Boschiroli, M.L., S. Ouahrani-Bettache, V. Foulongne, S. Michaux-Charachon and G. Bourg et al., 2002. The brucella suis virB operon is induced intracellularly in macrophages. Proc. Natl. Acad. Sci. USA., 99: 1544-1549.

Delrue, R.M., M. Martinez-Lorenzo, P. Lestrate, I. Danese and V. Bielarz et al., 2001. Identification of Brucella sp. genes involved in intracellular trafficking. Cell Microbiol., 3: 487-497.

Guerra, H., 2007. The brucellae and their success as pathogens. Crit. Rev. Microbiol., 33: 325-331.

He, Y., S. Reichow, S. Ramamoorthy, X. Ding and R. Lathigra et al., 2006. Brucella melitensis triggers time-dependent modulation of apoptosis and downregulation of mitochondrion-associated gene expression in mouse macrophages. Infect Immun., 74: $5035-5046$.

Mikhael, M., S.F. Kim, M. Schranzhofer, S.S. Lin, A.D. Sheftel, E.W. Mullner and P. Ponka, 2006. Iron regulatory protein-independent regulation of ferritin synthesis by nitrogen monoxide. FEBS J., 273: 3828-3836.

Orino, K. and K. Watanabe, 2008. Molecular, physiological and clinical aspects of the iron storage protein ferritin. Vet. J., 178: 191-201.

O'Callaghan, D., C. Cazevieeille, A. Allardet-Servent, M.L. Boschiroli and G. Bourg et al., 1999. A homologue of the Agrobacterium tumefaciens VirB and Bordetella pertussis Ptl type IV secretion systems is essential for intracellular survival of Brucella suis. Mol. Microbiol., 33: $1210-1220$.

Pappas, G., P. Papadimitriou, N. Akritidis, L. Christou and E.V. Tsianos, 2006. The new global map of human brucellosis. Lancet Infect. Dis., 6: 91-99.

Pham, C.G., C. Bubici, F. Zazzeroni, S. Papa and J. Jones et al., 2004. Ferritin heavy chain upregulation by NF-êB inhibits $\mathrm{TNF} \alpha$-induced apoptosis by suppressing reactive oxygen species. Cell, 119: 529-542.

Rajashekara, G., L. Eskra, A. Mathison, E. Petersen, Q. Yu, J. Harms and G. Splitter, 2006. Brucella: Functional genomics and host-pathogen interactions. Anim. Health Res. Rev., 7: 1-11.

Robinson-Dunn, B., 2002. The microbiology laboratory's role in response to bioterrorism. Arch. Pathol. Lab. Med., 126: 291-294. 
Rolan, H.G., A.B. den Hartigh, M. KahlMcDonagh, T. Ficht, L.G. Adams and R.M. Tsolis, 2008. VirB12 is a serological marker of Brucella infection in experimental and natural hosts. Clin. Vaccine Immunol., 15: 208-21 4.

Rouot, B., M.T. Alvarez-Martinez, C. Marius, P. Menanteau and L. Guilloteau et al., 2003. Production of the type IV secretion system differs among Brucella species as revealed with VirB5- and VirB8specific antisera. Infect. Immunity, 71: 1075-1082.
Tsuji, Y., 2005. JunD activates transcription of the human ferritin $\mathrm{H}$ gene through an antioxidant response element during oxidative stress. Oncogene, 24: 7567-7578.

Tsuji, Y., E. Moran, S.V. Torti and F.M. Torti, 1999. Transcriptional regulation of the mouse ferritin $\mathrm{H}$ gene: Involvement of $\mathrm{p} 300 / \mathrm{CBP}$ adaptor proteins in FER-1 enhancer activity. J. Biol. Chem., 274: 7501-7507. 